Bio - grafía. Escritos sobre la Biología y su Enseñanza. ISSN 2027-1034

Edición Extraordinaria. p.p. 293 - 301

Memorias del IX Encuentro Nacional de Experiencias en Enseñanza de la Biología y la Educación Ambiental. IV Congreso Nacional de Investigación en Enseñanza de la Biología.

\title{
APROXIMACIÓN AL EST ADO DEL ARTE DE LA FORMACIÓN CONTINUA DOCENTE EN LA PRIMERA INFANCIA PARA LA EDUCACIÓN EN CIENCIAS
}

\author{
Valencia Valbuena Fredy Gregorio \\ Tuay Sigua Rosa Nidia ${ }^{\dagger}$
}

\section{RESUMEN}

A continuación, se presenta los avances de la revisión documental acerca del proceso de la formación continua de profesores de primera infancia en la educación en ciencias que hace parte de la propuesta de investigación doctoral "LA EDUCACIÓN EN CIENCIAS EN LA PRIMERA INFANCIA DESARROLLADA EN COMUNIDADES DE FORMACIÓN: UN ESTUDIO DE CASO. Se realizó una revisión documental, cuyos resultados evidencian el escaso alcance de las políticas de formación continua para profesoras de la primera infancia, así como el esfuerzo y las limitaciones que se presentan al momento de la educación en ciencias en estos niveles educativos.

\section{PALABRAS CLAVE:}

Formación continua docente, primera infancia y educación en ciencias

\section{ABSTRACT}

The Next, we present the advances of the documentary review on the process of continuing education of early childhood teachers in science education that is part of the doctoral research proposal "EDUCATION IN SCIENCES IN THE FIRST CHILD DEVELOPED IN COMMUNITIES OF TRAINING: A CASE STUDY ". A documentary review was carried out, the results of which show the scarce reach of the politics of continuing education for early childhood teachers, as well as the effort and limitations that are presented at the time of science education at these educational levels.

\section{KEYWORDS:}

Continuing teacher education, early childhood and science education

\footnotetext{
*Estudiante del Doctorado Interinstitucional en Educación DIE-Universidad Pedagógica Nacional Docente de Preescolar y Básica Primaria - Colegio Centro Integral José María Córdoba IED. Secretaria de Educación del Distrito SED. correo: fvalencia@pedagogica.edu.co

+ Profesora de planta-Universidad Pedagógica Nacional. Directora del Grupo de Investigación: Educación en Ciencias, Ambiente y Diversidad (EDUCADIVERSO). Docente del Doctorado Interinstitucional en Educación correo: rtuay@pedagógica.edu.co
} 
Bio-grafía. Escritos sobre la Biología y su Enseñanza. ISSN 2027-1034

Edición Extraordinaria. p.p. 293 - 301

Memorias del IX Encuentro Nacional de Experiencias en Enseñanza de la Biología y la Educación Ambiental. IV Congreso Nacional de Investigación en Enseñanza de la Biología.

\section{INTRODUCCIÓN}

La formación del profesorado ha constituido una línea de investigación de gran reconocimiento en la educación en ciencias, prueba de ello son las publicaciones internacionales. Así, Perales y Cañal (2000) abordan el capítulo "La formación del profesorado en ejercicio" a partir del desarrollo profesional. De igual manera, se destaca el crecimiento profesional en el Handbook, editado por Beijaard, Meijer y MorineDershimer y Tillema (2005) en el capítulo "What Do We Mean by Career-Long Professional Growth and how Can We Get It?". Así mismo, el desarrollo profesional en el Hanbook editado por Fraser, Tobin., y McRobie (2012) con el capítulo "Teacher Education and Professional Development".

Por otra parte, en torno a la enseñanza de las ciencias y la formación del profesorado, Loughran, Berry y Mulhall (2012) describen este campo en el capitulo "Science teaching and teacher education". Seguidamente, en el Handbook editado por Corrigan, Gunstone y Jones (2013) enfatizan la importancia del aprendizaje de los profesores de ciencias tanto en la formación inicial y continua en el capítulo "Formative Assessment in Learning to Teach Science".

En cuanto a la educación en ciencias en la primera infancia, Simon y Campbell (2012) destacan las principales líneas de investigación en el Second International Handbook of Science Education. En la misma perspectiva, Cabe y Sackes (2015) describen el estado de la investigación en el Handbook "Research in Early Childhood Science Education".

Además, se presentan investigaciones en la enseñanza de las ciencias en la primera publicadas en revistas especializadas en infancia como Early Childhood Education Journal, Topics in Early Childhood Special Education, Research in Early Childhood Science Education, Early Education and Development, International Journal of Early Childhood, Early Childhood Research Quarterly, Early Years, Child Development Perspectives, Advances in Early Education and Day Care, International Journal of Science Education y Early Child Development and Care, entre otras.

Con el panorama anterior se evidencia el creciente interés investigativo en la formación continua docente y/o el desarrollo profesional docente, en particular en la primera infancia, a nivel internacional, como lo refieren el gran número de investigaciones en Estados Unidos. Sin embargo, son escasas las menciones a este campo de investigación en el ámbito latinoamericano e hispano (UNESCO, 2016; Observatorio Internacional de la Profesión Docente, 2015).

\section{METODOLOGIA}

El siguiente marco de referencia ha sido construido a partir de la revisión documental del estado del arte sobre la formación continua de profesores en la primera infancia y la educación en ciencias en la primera infancia. El estudio se centró en los resultados de investigaciones reportadas en los Handbook así como en las bases de datos Scopus, 
Bio - grafía. Escritos sobre la Biología y su Enseñanza. ISSN 2027-1034

Edición Extraordinaria. p.p. 293 - 301

Memorias del IX Encuentro Nacional de Experiencias en Enseñanza de la Biología y la Educación Ambiental. IV Congreso Nacional de Investigación en Enseñanza de la Biología.

Web Science, Ebsco, Science Direct, TDR, Jstor, Redalyc, Scielo, Latindex, Dialnet y, en especial, artículos publicados en revistas especializadas en el campo de la formación continua y la educación en ciencias en la primera infancia. Los artículos ofrecen un panorama mundial, latinoamericano y nacional de la problemática a investigar. Los criterios de búsqueda fueron temporales, contemplando los últimos veinte años y la referencia a palabras claves de formación continua + primera infancia y educación en ciencias + primera infancia.

\section{RESULTADOS Y DISCUSIÓN}

\section{POLITICAS EDUCATIVAS PARA LA FORMACIÓN CONTINUA DOCENTE EN LA PRIMERA INFANCIA}

En los últimos años, organismos internacionales consideran indispensable proponer acciones que fomenten la calidad educativa a través de la legislación docente (UNESCO, 2016; OIT y UNESCO, 2008). Esto se constituye en un asunto que afecta, por supuesto, tanto al profesor como la calidad educativa. Así, la UNESCO (2016, p, 100) enuncia: "Como consecuencia de la falta de información, es esperable que la oferta existente incluya duplicidades y vacíos, a la vez programas de baja calidad, sin responder a las necesidades de formación continua de las docentes de primera infancia".

En el caso de Colombia, se considera que intervenir en la primera infancia desde el sistema educativo genera mayores competencias para potenciar, a futuro, una economía basada en el conocimiento (PND, 2015), además tiene efectos positivos en el desarrollo cognitivo y socioeconómico en la vida adulta (CPC, 2016). Sin embargo, otra es la realidad, aunque existe la Estrategia Nacional de Atención Integral a la Primera Infancia (AIPI)-Ley 151 "De Cero a Siempre" para 4.6 millones de niños y niñas de 0 a cinco años, solamente hay cobertura para 1.051.005 niños y niñas, que están en su mayoría en los sectores con más alta vulnerabilidad social (CPC, 2015).

El anterior escenario deja al descubierto la necesidad de un proyecto de formación continua de profesores de primera infancia y se dé alcance al indicador de agentes educativos cualificados. Todo esto sustentado en las bajas competencias pedagógicas que tiene el personal que está en la $\mathrm{AEPI}$ en relación con los docentes de preescolar (OCDE, 2016). Así, las estadísticas mostraron la cualificación de 49.672 personas para 2014 y se espera superar, para 2018, las 110.000 personas que estarán en la AIPI (CPC, 2015).

Teniendo en cuenta estos aspectos, es prioritario que el Ministerio de Educación Nacional de Colombia (MEN), en trabajo conjunto con las instituciones formadoras de profesores en primera infancia (universidades, instituciones técnicas y otras), establezcan una ruta de acción formativa que den cumplimiento a la política pública educativa "De Cero a Siempre", además de vincular la exploración del medio en la educación inicial (MEN, 2014a) y la cualificación del talento humano (MEN, 2014b). 
Bio - grafía. Escritos sobre la Biología y su Enseñanza. ISSN 2027-1034

Edición Extraordinaria. p.p. 293 - 301

Memorias del IX Encuentro Nacional de Experiencias en Enseñanza de la Biología y la Educación Ambiental. IV Congreso Nacional de Investigación en Enseñanza de la Biología.

\section{LA FORMACIÓN CONTINUA DOCENTE EN LA PRIMERA INFANCIA Y LA EDUCACIÓN EN CIENCIAS}

En los últimos años se ha configurado la necesidad de la formación continua docente de los profesores de la primera infancia, pero especialmente se ha debatido sobre la importancia que la educación en ciencias sea parte de este proyecto de cualificación profesional. En este orden, desde la formación continua docente (FCD), a nivel general, McLeod (2015) propone el mejoramiento de la práctica de los profesores desde la reflexión crítica, teniendo en cuenta los intereses de los niños, las exigencias de la educación moderna y la configuración de redes, colectivos o comunidades de docentes. lgualmente, Swaminathan, Byrd, Humphrey, Heinsch y Mitchell (2013) destacan el impacto efectivo de los programas de desarrollo profesional en docentes de preescolar y su correspondencia en la promoción de resultados positivos para los estudiantes.

En cuanto a la FCD, pero desde las ciencias, Bryce, Wilmes y Bellino (2016) afirman que constituir grupos de profesores favorece el desarrollo de la identidad a través del desarrollo profesional, siendo el diálogo cogenerativo y las comunidades profesionales las que promueven la formación y la colaboración para la enseñanza de las ciencias. De igual modo, Miller, Curwen, White-Smith y Calfee (2014) reafirman la necesidad de diseñar programas de desarrollo profesional a partir de la colaboración continua entre la universidad y la escuela, para que los profesores de kínder, primero y segundo puedan contrarrestar factores que afectan la enseñanza de las ciencias.

\section{LA EDUCACIÓN EN CIENCIAS EN LA PRIMERA INFANCIA DESDE LAS PRÁCTICAS INSTIUCIONALES}

Indagando por los resultados de investigaciones el interés de la primera infancia está centrado en otras áreas del conocimiento como el lenguaje, las artes, las matemáticas y la lúdica, dejando de lado la comprensión de los fenómenos naturales que deben ser un propósito de enseñanza en la primera infancia. Al respecto, se plantea que, en la primera infancia, los niños ingresan a la institución con gran capacidad de aprendizaje, en particular para las ciencias y se debate la creencia que los niños son pensadores abstractos y simplistas: de hecho, alcanzan potentes aprendizajes de la ciencia porque ya tienen un conocimiento del mundo natural. Así mismo, desarrollan ideas explicativas en dominios específicos (biología, física, química, y la psicología), y configuran componentes universales compartidos sin importar el origen cultural, social y económico (National Academy of Science, 2007).

Entonces, armonizar la forma en cómo los niños aprenden ciencia con la manera de enseñar la ciencia es un aspecto clave; de ahí la importancia de la formación de los docentes que orientan la Educación en Ciencias en la Primera Infancia (ECPI). En ello, 
Bio - grafía. Escritos sobre la Biología y su Enseñanza. ISSN 2027-1034

Edición Extraordinaria. p.p. 293 - 301

Memorias del IX Encuentro Nacional de Experiencias en Enseñanza de la Biología y la Educación Ambiental. IV Congreso Nacional de Investigación en Enseñanza de la Biología.

Birbili (2007) plantea la necesidad de tener marcos conceptuales para la enseñanza de las ciencias, e invita a los profesores de primera infancia a organizar la enseñanza y el aprendizaje alejados de los hechos inertes.

Al mismo tiempo, Ginsburg y Golbeck (2004) consideran necesario que los maestros desarrollen planes de estudios creativos y agradables a partir de proyectos, además de identificar los intereses de los niños, referidos a su nivel socioeconómico analizando, y de los aportes que pueden tener los padres al vincularse con la educación.

Sobre la experiencia de los profesores de primera infancia, Saçkes (2014) evidencia que los cursos que hacen los profesores, los materiales dispuestos en el aula y las percepciones sobre la capacidad del niño para el aprendizaje son elementos que influyen para que los docentes enseñen conceptos particulares de las ciencias. De igual modo, Cabe y Sackes (2015) plantean que existen por lo menos 16 líneas de investigación que configuran un panorama general de las tendencias en la educación en ciencias en la primera infancia, pero se evidencia la falta de relación con la formación continua de los profesores.

Este panorama plantea contrastes en la manera cómo se asume la enseñanza de las ciencias en la primera infancia. Por un lado, creyendo que la actividad del docente es descubrir, explicar y codificar principios generales de enseñanza de algunas disciplinas que no está al alcance de los niños. Por otro lado, está la mirada diferenciadora, que permite construir escenarios futuros para generar conocimientos fundamentales en la configuración de un pensamiento científico en los niños.

\section{LOS DOCENTES DE PRIMERA INFANCIA EN LA FORMACIÓN CONTINUA}

Las investigaciones sobre la formación de los profesores de primera infancia para la educación en ciencias consideran que los niños pequeños han expresado la falta de enseñanza de las ciencias, lo que está relacionado con el conocimiento didáctico del contenido (PCK) de los profesores (Hong, Torquati y Molfese, 2013).

En cuanto a las actitudes y creencias en la enseñanza de las ciencias de profesores de preescolar, Maier, Greenfield y Bulotsky-Shearer (2013) analizan la influencia que tiene el origen del maestro, el nivel de educación y los años de experiencia para realizar acciones de enseñanza de las ciencias. En la misma dirección, Pendergast, Lieberman-Betz y Vail (2015) recomiendan que se investigue el impacto del desarrollo profesional en la enseñanza de la ciencia, ya que los profesores de preescolar la consideran difícil para enseñar y muy abstracta para los niños, además las experiencias personales de los profesores (que, por lo general, no son positivas).

Sumado a las dificultades, Miller, Curwen, White-Smith y Calfee (2014) consideran que, entre las falencias de los conocimientos en ciencias en los profesores, está la falta de tiempo para la enseñanza de las ciencias, porque se priorizan otras áreas como el lenguaje y las matemáticas. 
Bio - grafía. Escritos sobre la Biología y su Enseñanza. ISSN 2027-1034

Edición Extraordinaria. p.p. 293 - 301

Memorias del IX Encuentro Nacional de Experiencias en Enseñanza de la Biología y la Educación Ambiental. IV Congreso Nacional de Investigación en Enseñanza de la Biología.

Este panorama permite visualizar las dificultades que enfrentan los profesores de primera infancia para educar en ciencias: algunas son impuestas por el mismo profesor, al creer que no son capaces de enseñar ciencias; otras son producidas por las experiencias negativas que han tenido para educar en ciencias, y también las concepciones de la psicología cognitiva, en donde consideran que los niños aún no están preparados para las ciencias y también se denotan factores externos como el tiempo asignado para el desarrollo del currículo en ciencias.

\section{CONCLUSIONES}

A pesar de los esfuerzos para enseñar ciencias en los grados iniciales, los profesores de primera infancia están lejanos por diversas situaciones, como la escasa formación disciplinar, las experiencias profesionales, los tiempos institucionales, el cumplimiento en la enseñanza de otras áreas del conocimiento, el desinterés personal, entre otros factores que han impedido una formación profesional estructurada para educar en ciencias. Entonces, considerando que ya están diseñadas las políticas de formación continua para la primera infancia desde lineamientos tanto nacionales como por parte de organismos internacionales, es necesario ir vinculando y reestructurando estas políticas docentes y, a su vez, interconectarlas con la educación en ciencias en la primera infancia.

Por otro lado, los cambios constantes en la sociedad del conocimiento y el papel que ocupa la educación exigen la reorganización de los procesos de formación continua de los educadores de la primera infancia, que permita una educación de calidad cercana a la realidad, coherente con las culturas y poblaciones regionales. Así, se busca en los procesos de formación continua que el maestro esté preparado para abordar los conocimientos que tiene que ofrecer a sus estudiantes mientras obtiene consejos y ejemplos de cómo estructurar las experiencias de aprendizaje y las iniciativas de enseñanza (Tuay-Sigua, Giordano, Testa, 2017).

En general, el campo de la formación continua para educar en ciencias en la primera infancia es una línea de investigación esencial y pertinente que puede contribuir desde la profesión docente y el mejoramiento de la calidad educativa. 
Bio - grafía. Escritos sobre la Biología y su Enseñanza. ISSN 2027-1034

Edición Extraordinaria. p.p. 293 - 301

Memorias del IX Encuentro Nacional de Experiencias en Enseñanza de la Biología y la Educación Ambiental. IV Congreso Nacional de Investigación en Enseñanza de la Biología.

\section{REFERENCIAS BIBLIOGRÁFICAS}

Birbili, M. (2007). Making the case for a conceptually based curriculum in early childhood education. Early Childhood Education Journal, 35(2), 141-147. DOI: $10.1007 / \mathrm{s} 10643-006-0112-0$

Beijaard, D., Meijer, P., Morine-Dershimer, G., y Tillema, H (2005). Teacher Professional Development in Changing Conditions. Netherlands: Springer

Bryce, N., Wilmes, S y Bellino, M. (2016). Inquiry identity and science teacher professional development. Cultural Studies of Science Education. Cultural Studies of Science Education, 11(2), 235-251. DOI: 10.1007/s11422-015-9725-1

Cabe, K ., y Sackes, M. (Eds.). (2015). Research in Early Childhood Science Education. New York: Springer.

Consejo Privado de Competitividad (CPC). (2016). Informe Nacional de Competitividad 2016-2017. Colombia. (2015). Informe Nacional de Competitividad 2016-2017. Colombia.

Corrigan, D., Gunstone, R., y Jones, A. (eds). (2013). Valuing Assessment in Science Education: Pedagogy, Curriculum, Policy. New York-London: Springer.

Frase, B., Tobin, K., y McRobbie, C. (eds). (2012). Second International Handbook of Science Education. Vol 1. London-New York: Springer

Ginsburg, H., y Golbeck, S. (2004). Thoughts on the future of research on mathematics and science learning and education. Early Childhood Research Quarterly, 19(1). DOI: 10.1016/j.ecresq.2004.01.013

Hong, S., Torquati, J y Molfese, V. (2013). Theory guided professional development in early childhood science education. Advances in Early Education and Day Care, 17, 1-3. DOI: $10.1108 / S 0270-4021(2013) 0000017005$

Loughran, J., Berry, A., y Mulhall, P. (2012). Understanding and Developing Science Teachers' Pedagogical Content Knowledge. (2a edition). Rotterdam: Sense Publishers.

Maier, M., Greenfield, D., y Bulotsky-Shearer, R. (2013). Development and validation of a preschool teachers' attitudes and beliefs toward science teaching questionnaire. Early Childhood Research Quarterly, 28(2), 366-378. DOI: 10.1016/j.ecresq.2012.09.003

McLeod, N. (2015). Reflecting on reflection: improving teachers' readiness to facilitate participatory learning with young children. Professional Development in Education, 41(2), 254-272. DOI: 10.1080/19415257.2013.805306 
Bio-grafía. Escritos sobre la Biología y su Enseñanza. ISSN 2027-1034

Edición Extraordinaria. p.p. 293 - 301

Memorias del IX Encuentro Nacional de Experiencias en Enseñanza de la Biología y la Educación Ambiental. IV Congreso Nacional de Investigación en Enseñanza de la Biología.

Miller, R., Curwen, M., White-Smith, K., y Calfee, R. (2014). Cultivating Primary Students' Scientific Thinking Through Sustained Teacher Professional Development. Early Childhood Education Journal, 43(4). DOI: 10.1007/s10643-014-0656-3

Ministerio de Educación Nacional-MEN. (2014a). La exploración del medio en la educación inicial. Documento ํㅜ 24. Serie de orientaciones pedagógicas para la educación inicial en el marco de la atención integral. República de Colombia.

(2014b). Cualificación del talento humano que trabaja con primera infancia. Documento no 19. Referentes técnicos para la cualificación del talento humano que trabaja con primera infancia. República de Colombia.

National Academy of Science. (2007). Taking Science to School: Learning and Teaching Science in Grades K-8. Committee on Science Learning, Kindergarten Through Eighth Grade. Board on Science Education, Center for Education. Division of Behavioral and Social Sciences and Education. Washington, DC: The National Academies. Recuperado de Presshttp://nap.edu/11625. DOI 10.17226/11625

Observatorio Internacional de la Profesión Docente. (2015). Archivos sobre formación permanente. Docentes en educación infantil. Recuperado de http://www.ub.edu/obipd/formacion-permanente/infantil-formacion-permanente/

OCDE. (2016). La educación en Colombia. Revisión de políticas nacionales de educación.

OIT/UNESCO. (2008). Recomendación conjunta de la OIT y la UNESCO relativa a la situación del personal docente (1966) y recomendación de la UNESCO relativa a la condición del personal docente de enseñanza superior (1997)

Pendergast, E., Lieberman-Betz, R., y Vail, C. (2015). Attitudes and Beliefs of Prekindergarten Teachers Toward Teaching Science to Young Children. Early Childhood Education Journal. DOI: 10.1007/s 10643-015-0761-y

Perales, F y Cañal, P. (2000). Didáctica de las ciencias experimentales. Barcelona: Marfil

Plan Nacional de Desarrollo (PND) 2014-2018. (2015). Todos por un nuevo país. Departamento Nacional de Planeación. República de Colombia.

Saçkes, M. (2014). How often do early childhood teachers teach science concepts? Determinants of the frequency of science teaching in kindergarten. European Early Childhood Education Research Journal, 22(2), 169-184. doi:10.1080/1350293X.2012.704305

Simon, S., y Campbell, S. (2012). Teacher Learning and Professional Development in Science Education. En Fraser, B.; Tobin, K. y Campbell, M. (eds). Second International Handbook of Science Education. 307-321. New York.

Swaminathan, S., Byrd, S., Humphrey, C., Heinsch, M., y Mitchell, M. (2013). Winning Beginnings Learning Circles: Outcomes from a Three-Year School Readiness Pilot. Early Childhood Education Journal, 42(4). DOI: 10.1007/s 10643-013-0606-5 
Bio - grafía. Escritos sobre la Biología y su Enseñanza. ISSN 2027-1034

Edición Extraordinaria. p.p. 293 - 301

Memorias del IX Encuentro Nacional de Experiencias en Enseñanza de la Biología y la Educación Ambiental. IV Congreso Nacional de Investigación en Enseñanza de la Biología.

Tuay-Sigua, R., Giordano, E., y Testa (2017). El sentido de hacer ciencia con los niños. En Enseñanza de las Ciencia e Infancia. Problemáticas y avances de teoría y campo desde lberoamérica. Santiago de Chile: Universidad Católica de Chile. En prensa.

UNESCO. (2016). Estrategia Regional sobre Docentes. Estado del arte y criterios orientadores para la elaboración de políticas de formación y desarrollo profesional de docentes de primera infancia en América Latina y el Caribe. Santiago de Chile. 